\title{
Investment Without Regulatory Commitment: The Case of Elastic Demand
}

\author{
SANTIAGO URBIZTONDO \\ Universidad de San Andrés \\ Instituto Torcuato Di Tella and Universidad Nacional de La Plata, Argentina
}

\begin{abstract}
This paper analyzes a regulation game with asymmetric information and lack of commitment. It expands Besanko and Spulber's (1989) framework to the case of elastic demands using a generalized Nash solution. It is found that the most important property of the equilibrium with inelastic demand is not carried over to the elastic demand case, i.e., incomplete information worsens underinvestment, contrasting to Besanko and Spulber's result.
\end{abstract}

\section{Introduction}

It is widely recognized that long-term relationships in which parties cannot commit to future actions can result in inefficiencies due to "opportunism." (See Kydland and Prescott (1977, 619-637) and Williamson (1975).) In the case of a relationship between a firm and a regulator, the lack of the regulator's commitment to set prices that allow the firm to recover the full amount of sunk investment results in lower-than-optimal levels of investment. On the other hand, introduction of asymmetric information in regulatory games has captured significant amount of research. (See the pioneering work of Baron and Myerson (1982, 911-930).) Furthermore, solutions to dynamic problems also recognize as crucial the ability to commit not to use information revealed throughout the game. (See Laffont and Tirole (1988, 1153-1175) and Baron and Besanko (1987, 413-436).)

In a recent paper, Besanko and Spulber (1989) analyze a regulatory environment, with the regulated firm possessing private information about its cost. ${ }^{1}$ The structure of their model is as follows: the firm observes a realization of a cost parameter $(\theta)$ and then chooses a level of investment $(k)$; the regulator does not observe $\theta$ but observes $k$, which allows him to infer $\theta$ and set the price accordingly, given that he is unable to respect any previous agreement with the firm. ${ }^{2}$ The demand function and the level of investment are observed by both parties without incurring any cost. Limiting the study to the case of a perfectly inelastic demand

1 A modified version of their paper is Besanko and Spulber (1992).

2 As it is the case in screening models with commitment, in which the timing is basically reversed. See Baron and Myerson (1982). 
function, they find that the regulator is able to separate fully the different types of firms. The regulator is seen as offering a price schedule to the firm satisfying the condition that the information revealed by the firm when choosing an investment level does not induce the regulator to change its initial offer. Among other findings, they conclude that asymmetric information alleviates underinvestment and that it can even happen that the less efficient firms overinvest, as those are the firms that need to invest more to signal their low state of technology.

This paper extends Besanko and Spulber's model and shows that the effect of asymmetric information upon the level of investment depends on the elasticity of demand faced by the regulated firm. I accordingly let the demand function be elastic (with a constant elasticity greater than one). The equilibrium is also separating. Incomplete information, though, worsens underinvestment for the least efficient firms. This result is due to the fact that for elasticities greater than one investment is a decreasing function of the technology parameter $\theta$, for which the inefficient firms have to invest less than in the complete information case, so that the incentive compatibility constraint of the more efficient firms is satisfied.

\section{The Model}

Let the demand function be given by

$$
x=D(p)=p^{-\varepsilon}
$$

where $x$ represents quantity, $p$ is the price, and $\varepsilon$ is the demand elasticity. An exogenously given firm is scheduled to provide the whole market. Following Besanko and Spulber, the regulated firm has a cost function

$$
C(x, k, \theta)=\frac{x^{2} \theta}{2 k}+r k
$$

where $k$ is the level of investment, $r$ is the rental cost of capital, and $\theta$ is the efficiency parameter of the firm $\left(\theta \in\left[\theta_{0}, \theta_{1}\right]\right)$, i.e., the $\theta_{0}$ firm is the most efficient one. The term $x^{2} \theta / 2 k$ corresponds to operating costs, which are decreasing on the level of investment. Both the firm and the regulator know $D(p)$, while $r$ and $k$ are also public knowledge. The state of nature $\theta$ is observed only by the firm. Consumer surplus is given by $S(p)=\int_{p}^{\infty} D(z) d z$. The regulator chooses a price $p$ and a transfer $T$ in order to maximize the Generalized Nash welfare function: ${ }^{4}$

$$
W(p, T)=[S(p)-T]^{\alpha}\left[T+p D(p)-\frac{(D(p))^{2} \theta}{2 k}-s k\right]^{2-\alpha} .
$$

3 This is also the case in Besanko and Spulber's (1989) paper, although, since they consider an inelastic demand function, the consumer surplus (and, indeed, the whole problem) is only defined by introduction of a consumers' maximum willingness to pay for the outcome.

4 To make the result of this paper contrast with that of Besanko and Spulber (1989) solely due to the elasticity of demand, I chose to use the same welfare function with a minor additional variable $\alpha$ ), which Besanko and Spulber consider (implicitly) equal to one. 
The first bracket represents the consumer surplus net of transfers; the second is the firm's profit; $^{5}$ and $0<\alpha \leq 2$ is a weighing parameter (i.e., $\alpha=0$ represents profit maximization).

The timing of the model is as follows: the firm observes a realization of $\theta$; next the regulator offers a schedule $p(k), T(k)$; and finally the firm chooses $k$ as a function of the realization $\theta$ and the schedule offered by the regulator.

At a separating equilibrium, the regulator is able to infer $\theta$ from $k$. Knowing this and the fact that the regulator is unable to commit to this schedule after discovering $\theta$, the firm will choose $k$ based on $\theta$ and the price and transfer that the regulator will set after calculating $\theta$ from $k$.

\section{Investment Under Complete Information}

When regulatory commitment is feasible and $\theta$ is commonly known, the regulator will set $p$ and $T$ in such a way that price equals marginal cost and the level of capital is the level that minimizes the cost of producing $x$. This solution can be implemented following Loeb and Magat (1979, 399-404), by giving all the (gross) consumer surplus to the firm and redistributing part of it to the consumers through a lump-sum tax on the firm. In this case, $p=x \theta / k$ and $k=\left(x^{2} \theta / 2 r\right)^{1 / 2}$. Combining these two equations with equation (1) results in

$$
\hat{p}=(2 \theta r)^{\frac{1}{2}}
$$

and

$$
\hat{k}=(2 r)^{-\frac{(1+\varepsilon)}{2}} \theta^{\frac{1-\varepsilon}{2}} \text {. }
$$

In the absence of regulatory commitment, on the other hand, the firm maximizes its profits subject to the price and transfer arising from the maximization of (3) with respect to $p$ and $T$. Using the first-order conditions in the maximization of (3) and noting that $S^{\prime}(p)=-D(p)$, we obtain that $p=x \theta / k$ and $T=S(p)((2 \alpha) / 2)+\left[x^{2} \theta / 2 k+s k-p x\right]$, which, after using equation (1) and noting that $S(p)=p^{-(\varepsilon-1)} /(\varepsilon-1)$ can be solved together resulting in

$$
\tilde{p}=\left(\frac{\theta}{k}\right)^{\frac{1}{(1+\varepsilon)}},
$$

and

$$
\tilde{T}=\left[\frac{4-\alpha-\alpha \varepsilon}{4(\varepsilon-1)}\right]\left(\frac{\theta}{k}\right)^{\frac{1-\varepsilon}{1+\varepsilon}}+\frac{\alpha s k}{2}
$$

5 Observe that the regulator considers as rental cost of capital only the scrap value of capital, $s$, where $s \leq r$, with $s<r$ corresponding to the case where some investment costs are sunk. This characteristic just shows the regulator's lack of commitment and opportunistic behavior after the investment decision was done, i.e., he only considers the opportunity cost of capital ex-post of the investment decision. 
The firm's problem is then to $\max \Pi=T+p D(p)-(D(p))^{2} \theta / 2 k-r k$ with respect to $k$, subject to equations (1), (6) and (7). After introducing these equations into $\Pi$ and solving for $k$ we obtain

$$
\kappa=\left(\frac{2(2 r-s)}{2-\alpha}\right)^{-\frac{(1+\varepsilon)}{2}} \theta^{\frac{1-\varepsilon}{2}} .
$$

Comparing $\tilde{k}$ in (8) and $\hat{k}$ in (5), we obtain that, for $\alpha=1, \hat{k} \leq \hat{k}$, with strict inequality for $s<r$, that is, underinvestment results from lack of commitment. Also, from (8), we obtain the expected result that the level of investment is a decreasing function of the weight asigned by the regulator to the firm's profits, i.e., $\partial \tilde{k} / \partial \alpha<0$.

\section{Investment Under Incomplete Information ${ }^{6}$}

Let $g(\theta)$ be the regulator's prior beliefs about $\theta$, which are updated after observing $k$. Let $\delta(k)$ be the expected value of $\theta$ after observing $k^{7}$

Definition: A sequential equilibrium for the regulation game consists of the strategies $k^{*}(\theta), p^{*}(k)$, and $T^{*}(k)$, and beliefs $\delta^{*}(k)$ such that:

(A) The firm chooses $k^{*}(\theta)$ to

$$
\max \Pi\left(k, p^{*}, T^{*}, \delta^{*}\right)=T^{*}+p^{*} D\left(p^{*}\right)-\left(D\left(p^{*}\right)\right)^{2} \frac{\theta}{2 k-r k} .
$$

(B) The regulator chooses $p^{*}(k)$ and $T^{*}(k)$ to

$$
\max W\left(k, p, T, \delta^{*}\right)=(S(p)-T)\left(T+p D(p)-(D(p))^{2} \frac{\delta^{*}(k)}{2 k-s k}\right) .
$$

(C) The regulator's beliefs on the equilibrium path, $k^{*-1}(k) \neq \phi,{ }^{8}$ are consistent with Bayes' rule and the firm's equilibrium strategy $k^{*}(\theta)$.

Note that with incomplete information a separating equilibrium has to respect the incentive compatibility constraint given by

$$
\Pi\left(\theta, k^{*}(\theta)\right) \geq \Pi\left(\theta, k^{*}(\tilde{\theta}) \text { for all } \theta \text { and } \tilde{\theta} \in\left[\theta_{0}, \theta_{1}\right] .^{9}\right.
$$

6 To simplify the algebra, I consider $\alpha=1$ in this section.

7 It becomes obvious later that the prior $g(\theta)$, which plays a crucial role in screening models with commitment as Baron and Myerson's (1982), is totally irrelevant for the (separating) equilibrium without commitment.

8 That is, excluding observed levels of investment $(k)$ which cannot be the result of an optimal strategy $\left(k^{*}\right)$ followed by any type of firm.

9 The individual rationality constraint is always satisfied by construction of the welfare function as long as the project is desirable, which is assumed to be the case. 
In what follows, I first show the difficulty one faces when trying to characterize the equilibrium, and then I identify two particularities of the equilibrium: incomplete information worsens underinvestment and the incentive compatibility constraint binds in only one direction. These two properties are illustrated in a discrete treatment of the equilibrium.

\subsection{Trying to Characterize the Equilibrium}

When the demand function is given by (1), the firm's problem can be written as maximize $\Pi(\theta, k)=T(k)+p(k)^{1-\varepsilon}-p(k)^{-2 \varepsilon} \theta / 2 k-r k$ with respect to $k$. Denoting $p^{\prime}(k)$ and $T^{\prime}(k)$ the first derivatives of the price and transfer functions, the first order condition for a maximum is

$$
T^{\prime}(k)+(1-\varepsilon) p(k)^{\varepsilon} p^{\prime}(k)+\frac{\varepsilon \theta p^{\prime}(k) p(k)^{-(2 \varepsilon+1)}}{k}+\frac{\theta p(k)^{-2 \varepsilon}}{2 k^{2}}=r
$$

from which we obtain

$$
\theta=\frac{\left(r-T^{\prime}(k)-(1-\varepsilon) p(k)^{-\varepsilon} p^{\prime}(k)\right)}{\left(\frac{\varepsilon p^{-(2 \varepsilon+1)} p^{\prime}(k)}{k}+\frac{p(k)^{-2 \varepsilon}}{2 k^{2}}\right)}
$$

In equilibrium, $\delta(k)=\theta$, so we can replace (10) into (6) and (7) to obtain $p(k)$ and $T(k)$ under incomplete information. Replacing (10) into (6) and after a few algebraic steps, $p^{\prime}(k)=\left(r-T^{\prime}(k)\right) p(k)^{\varepsilon}-p(k) / 2 k$.

Also, from equations (6) and (7), we obtain $T(k)=[(3-\varepsilon) / 4(\varepsilon-1)] p(k)^{\varepsilon-1}+s k / 2$. Then, $T^{\prime}(k)=[(3-\varepsilon) / 4] p(k)^{\varepsilon} p^{\prime}(k)+s / 2$ can be replaced into $p^{\prime}(k)$ to obtain the differential equation in $p(k)$ below

$$
p^{\prime}(k)=\frac{2(2 r-s) p(k)^{\varepsilon}-2 p(k) k^{-1}}{\left(4+(3-\varepsilon) p(k)^{2 \varepsilon}\right)},
$$

whose solution would characterize the equilibrium up to a constant of integration. ${ }^{10}$ Obviously, the difficulty found in characterizing the equilibrium consists of the difficulty of solving this differential equation. Nevertheless, certain (important) properties of the equilibrium are identified below. ${ }^{11}$

\subsection{Firms Underinvest Relative to Complete Information}

Recall investment under complete information and lack of commitment is a decreasing function of the technology parameter $\theta$ (i.e., $\partial \bar{k} / \partial \theta<0$ ), that is, efficient firms invest more. Therefore, $\delta^{\prime}(k)<0$ is a sensible condition to impose on the beliefs of the regulator. Note also that $p^{*}(\delta(k))$ and $T^{*}(\delta(k))$ are given by the adaptation to incomplete information of equations (6) and (7) below,

10 This constant of integration can be assigned a particular value using the universal divinity criterium (which basically requires that positive probability be assigned only to those types that would benefit the most from deviation). See Besanko and Spulber (1989), Cho and Kreps (1987), and note 16.

11 It is assumed that a separating equilibrium does in fact exist. See note 12 for a formalization of this assumption. 


$$
p^{*}=\left(\frac{\delta(k)}{k}\right)^{\frac{1}{(1+\varepsilon)}}
$$

and

$$
T^{*}=\left(\frac{3-\varepsilon}{4(\varepsilon-1)}\right)\left(\frac{\delta(k)}{k}\right)^{\frac{1-\varepsilon}{1+\varepsilon}}+\frac{s k}{2} .
$$

Replacing (11) and (12) into the profit function of the firm, and after rearranging terms,

$$
\Pi(\theta, p(\delta), T(\delta), \delta(k))=\left(\frac{\delta(k)}{k}\right)^{\frac{1-\varepsilon}{1+\varepsilon}}\left(\frac{(3 \varepsilon-1)}{4(\varepsilon-1)}-\frac{\theta}{2 \delta(k)}\right)+\frac{(s-2 r)}{2} k .
$$

Differentiating with respect to $k$ and evaluating at $\delta(k)=\theta$, i.e., at the equilibrium,

$$
\frac{\partial \Pi}{\partial k}=\frac{1}{4}\left(\frac{\theta}{k}\right)^{\frac{1-\varepsilon}{1+\varepsilon}}\left(\frac{\delta^{\prime}(k)}{\theta}+\frac{1}{k}\right)+\frac{(s-2 r)}{2}=0
$$

is the first-order condition for a maximum. ${ }^{12}$ If $\delta^{\prime}(k)=0$, as with complete information, $\partial \Pi / \partial k=0$ occurs at $k^{*}(\theta)=\widetilde{k}(\theta)=[2(2 r-s)]^{-(1+\varepsilon) / 2} \theta^{(1-\varepsilon) / 2}$. Nevertheless, if $\delta^{\prime}(k)<0$, the sensible case with incomplete information, $\partial \Pi / \partial k<0$ at $\widetilde{k}(\theta)$, which means that $k^{*}(\theta)<\bar{k}(\theta){ }^{13}$

\subsection{The Incentive Compatibility Constraint (ICC) Only Binds in One Direction}

To see this, consider the following question: would a firm $\theta_{i}$ want to be considered as a $\theta_{j}$ firm if the cost of doing so is to mimic the level of investment of the $j$ firm?

Let $\Pi\left(\widetilde{k}\left(\theta_{j}\right), \theta_{i}\right)$ denote the profit of a $\theta_{i}$ firm when it invests $\tilde{k}\left(\theta_{j}\right)$ so that the regulator believes its type is $\theta_{j}$. Then, using equations (1), (6) and (7), and regrouping terms,

and

$$
\Pi\left(\frac{\tilde{k}\left(\theta_{i}\right)}{\theta_{i}}\right)=\left(\frac{\theta_{i}}{\widetilde{k}\left(\theta_{i}\right)}\right)^{\frac{1-\varepsilon}{1+\varepsilon}}\left(\frac{\varepsilon+1}{4(\varepsilon-1)}\right)+\left(\frac{s}{2}-r\right) \tilde{k}\left(\theta_{i}\right)
$$

$$
\Pi\left(\frac{\widetilde{k}\left(\theta_{j}\right)}{\theta_{i}}\right)=\left(\frac{\theta_{j}}{\widetilde{k}\left(\theta_{j}\right)}\right)^{\frac{1-\varepsilon}{1+\varepsilon}}\left(\frac{3 \varepsilon-1}{4(\varepsilon-1)}-\frac{\theta_{i}}{2 \theta_{j}}\right)+\left(\frac{s}{2}-r\right) \widetilde{k}\left(\theta_{j}\right)
$$

12 The second-order condition for a maximum is given by $\partial^{2} \Pi / \partial k^{2}$ (evaluated at $\left.\delta(k)=\theta\right)<0$, where $\partial \Pi^{2 / \partial} k^{2}=-\left[\delta(k) / k J^{2 \varepsilon /(I+\varepsilon)}\left\{[(7 \varepsilon+1) / 4(\varepsilon+1)]\left(\delta^{\prime}(k)^{2} / k \delta(k)\right)+[(1-\varepsilon) /(1+\varepsilon)] \delta^{\prime}(k) / k^{2}+\delta^{\prime \prime}(k) / 2 k\right\}\right.$.

It is assumed that $\delta^{\prime}(k)$ and $\delta^{\prime \prime}(k)$ are such that this condition is satisfied. Note that if $\delta^{\prime \prime}(k) \geq 0, \delta^{\prime}(k)<0$ is sufficient to satisfy this condition, in which case a separating equilibrium does in fact exist.

13 In Besanko and Spulber (1989), $\partial \Pi / \partial k=s / 2-r+\theta / k^{2}-\delta(k) / 2 k^{2}+\delta^{\prime}(k) / 2 k$, which evaluated at $\delta(k)=\theta$, and since $\delta^{\prime}(k)>0$ when $\varepsilon=0$, results in $k(\theta)^{*}>(\theta)$. 
denote the profit of the firm of type $i$ when it invests the level of investment of its own type and when it mimics type $j$ 's level of investment under complete information, respectively. Furthermore, using equation (8) and after a few replacements, $\Pi\left(\mathcal{k}\left(\theta_{i}\right), \theta_{i}\right) \geq \Pi\left(\widetilde{k}\left(\theta_{j}\right), \theta_{i}\right)$ if and only if

$$
A=\left(\frac{\theta_{j}}{\theta_{i}}\right)^{\frac{\varepsilon-1}{2}}>1+(\varepsilon-1)\left(1-\frac{\theta_{i}}{\theta_{j}}\right)=B .
$$

Notice first that the equation above becomes an equality when $i=j$, i.e., $A=B$. Denote $\beta=\theta_{i} / \theta_{j}$ (such that $A=B$ for $\beta=1$ ). Therefore, if $\partial A / \partial \beta>\partial B / \partial \beta$ evaluated at $\beta \geq 1$, equation (13) holds for $i>j$, i.e., the less efficient firm does not want to imitate the most efficient one. Observing that $\partial A / \partial \beta=[(1-\varepsilon) / 2] \beta^{-(1+\varepsilon) / 2}<0$ and $\partial B / \partial \beta=1-\varepsilon<0$ for $\varepsilon>1$, we can see that this is indeed the case.

On the other hand, we have to see whether the equation (13) is not respected by the most efficient firm, which would indicate that the incentive compatibility constraint given in (9) binds only for the most efficient firms. I show below that this is the case for $i$ and $j$ sufficiently close to each other. To do so, denote $\beta^{\prime}=1 / \beta$, such that the equation (13) can be expressed as $A=\beta^{\prime(\varepsilon-1) / 2} \geq 1+(\varepsilon-1)\left(1-\beta^{\prime-1}\right)=B$. It is easy to see, then, that $\partial A / \partial \beta^{\prime}<\partial B / \partial \beta^{\prime}$ for $\beta^{\prime} \geq 1$ if and only if $\beta^{\prime(\varepsilon+1) / 2}<2$, meaning that for $\beta$ sufficiently close to one the ICC binds for $i<j$, i.e., the most efficient firm wants to imitate the less efficient one if the cost of doing so is to invest at the latter's complete information level of investment. ${ }^{14}$

Therefore, the more efficient firms have incentives to imitate (closely) less efficient ones, which means that the incentive compatibility constraint given by (9) binds in only one direction, i.e., for $\tilde{\theta}>\theta$.

\subsection{A Discrete Example}

This subsection makes use of subsection 4.3. to characterize the sequential equilibrium when $\theta$ takes only two values, $\theta_{0}$ or $\theta_{1}$, with $\theta_{1}>\theta_{0}$. From the discussion above, only the second of the two incentive compatibility constraints below is binding,

$$
\begin{aligned}
& \Pi\left(\frac{k^{*}\left(\theta_{1}\right)}{\theta_{1}}\right) \geq \Pi\left(\frac{k^{*}\left(\theta_{0}\right)}{\theta_{1}}\right), \\
& \Pi\left(\frac{k^{*}\left(\theta_{0}\right)}{\theta_{0}}\right) \geq \Pi\left(\frac{k^{*}\left(\theta_{1}\right)}{\theta_{0}}\right) .
\end{aligned}
$$

Furthermore, if the equilibrium is separating, $k^{*}\left(\theta_{0}\right)=\widetilde{k}\left(\theta_{0}\right)$, since the most efficient firm is never imitated by a less efficient one and therefore does not have to deviate from the complete information level of investment to signal its type.

14 Note that it might happen that neither constraint binds when there is a discrete number of types and the types are too different from each other. In this case, incomplete information has no effect on investment. I abstract from this case. Furthermore, as in Milgrom and Roberts (1982) for the case of limit pricing, the constraint is always binding when there is a continuum of firms. 
Finally, the beliefs that support the separating equilibrium are given by

$$
\delta^{*}(k)=\theta_{1} \text { if } k=k^{*}\left(\theta_{1}\right), \theta_{0} \text { otherwise. }^{15,16}
$$

Therefore, $k^{*}\left(\theta_{1}\right)$ is given by $\Pi\left(\theta_{0}, k^{*}\left(\theta_{0}\right)\right)=\Pi\left(\theta_{0}, k^{*}\left(\theta_{1}\right)\right),{ }^{17}$ which after replacement of equations (11), (12), and (14) results in

$$
\left(\frac{\varepsilon+1}{4(\varepsilon-1)}\right)\left(\frac{\theta_{0}}{k^{*}\left(\theta_{0)}\right.}\right)^{\frac{1-\varepsilon}{1+\varepsilon}}-\left(\frac{3 \varepsilon-1}{4(\varepsilon-1)}-\frac{\theta_{0}}{2 \theta_{1}}\right)\left(\frac{\theta_{1}}{k^{*}\left(\theta_{1}\right)}\right)^{\frac{1-\varepsilon}{1+\varepsilon}}+\left(\frac{s}{2}-r\right)\left(k^{*}\left(\theta_{0}\right)-k^{*}\left(\theta_{1}\right)\right)=0 .
$$

Summarizing, for $\theta \varepsilon \theta_{0}, \theta_{1}$, the (universally divine) sequential equilibrium involves $p^{*}$, $T^{*}$, and $\delta^{*}(k)$ given by equations (11), (12), and (14) respectively, whereas $k^{*}\left(\theta_{0}\right)=\widetilde{k}\left(\theta_{0}\right)=[2(2 r-s)]^{-(1+\varepsilon) / 2} \theta_{0}^{(1-\varepsilon) / 2}$ and $k^{*}\left(\theta_{1}\right)$ is implicitly given by equation (15).

For example, for $\theta_{0}=1, \theta_{1}=3 / 2, r=1, s=4 / 5$, and $\varepsilon=2, \pi\left(\theta_{0}\right)=k^{*}\left(\theta_{0}\right)=0.2689$, $\widetilde{k}\left(\theta_{1}\right)=0.2196$ and $k^{*}\left(\theta_{1}\right)=0.125$. It is easy to check that this is indeed an equilibrium (and that the incentive compatibility constraint does not bind for the firm with cost parameter $\theta_{1}$.

\subsection{An Approximation to the Continuous Case}

Lastly, we close this section by extending the characterization of the sequential equilibrium with two types to $n$ types. Notice that the equilibrium relationship in the previous subsection can be repeated between that of a firm with cost parameter $\theta_{1}$ and another firm characterized by $\theta_{2}$, where $\theta_{2}>\theta_{1}$, provided that the relationship between $k^{*}\left(\theta_{0}\right)$ and $k^{*}\left(\theta_{1}\right)$ is as before. ${ }^{18}$ The equilibrium below makes use of this property between any two contiguous types of firms.

Let $\theta \in \theta_{0}, \theta_{1}, \ldots, \theta_{n}$. Then, the (universally divine) sequential equilibrium involves $p^{*}(k)$ and $T^{*}(k)$ as before,

$$
\begin{aligned}
& \delta^{*}(k)= \begin{cases}\theta_{i} & \text { if } k=k^{*}\left(\theta_{i}\right), \text { for } i=1, \ldots, n, \\
\theta_{0} & \text { otherwise, }\end{cases} \\
& k^{*}\left(\theta_{0}\right)=\widetilde{k}\left(\theta_{0}\right)=[2(2 r-s)]^{\frac{(1+\varepsilon)}{2}} \theta_{0^{2}}^{\frac{1-\varepsilon}{2}}
\end{aligned}
$$

and $k^{*}\left(\theta_{1}\right)$ implicitly given by

15 Beliefs $\delta^{*}(k)=\theta_{1}$ if $k \leq k^{*}\left(\theta_{1}\right), \delta^{*}(k)=\theta_{0}$ otherwise, would work also.

16 Note that these beliefs respect the universal divinity criterium (see Cho and Kreps (1987). To see this, note that beliefs $\delta(k)=\theta_{1}$ if $k \leq k^{* *}\left(\theta_{1}\right), \delta(k)=\theta_{0}$ otherwise, where $k^{* * *}\left(\theta_{1}\right)<k^{*}\left(\theta_{1}\right)$, would support other separating equilibria. Nevertheless, these beliefs do not respect the universal divinity criterium for out of equilibrium beliefs, since the regulator believes $\delta\left(k^{*}\left(\theta_{1}\right)\right)=\theta_{0}$, which is not sensible as the firm with cost parameter $\theta_{0}$ would never, regardless of what the regulator believes it is, choose $k^{*}\left(\theta_{1}\right)$.

18 This is possible because the incentive compatibility constraint binds in only one direction. 


$$
\left(\frac{\varepsilon+1}{4(\varepsilon-1)}\right)\left(\frac{\theta_{i-1}}{k^{*}\left(\theta_{i-1}\right)}\right)^{\frac{1-\varepsilon}{1+\varepsilon}}-\left(\frac{3 \varepsilon-1}{4(\varepsilon-1)}-\frac{\theta_{i-1}}{2 \theta_{i}}\right)\left(\frac{\theta_{i}}{k^{*}\left(\theta_{i}\right)}\right)^{\frac{1-\varepsilon}{1+\varepsilon}}+\left(\frac{s}{2}-r\right)\left(k^{*}\left(\theta_{i-1}\right)-k^{*}\left(\theta_{i}\right)\right)=0
$$

Here again, as in the example in subsection 4.4 illustrated, underinvestment results for all but one $\left(\theta_{0}\right.$, the most efficient) firm.

\section{Conclusion}

This paper considers a one period regulatory game in which a firm possessing private information about its cost is regulated by a regulator who cannot commit not to use the information revealed after observing the firm's investment decision.

Besanko and Spulber (1989) characterize the separating equilibrium for an inelastic demand. In their framework, the extent of underinvestment resulting from lack of commitment is alleviated when there is asymmetric information, and overinvestment may result for the less efficient firms.

In this paper, I consider the case of an elastic demand. The equilibrium is also separating but incomplete information accentuates the underinvestment resulting from lack of commitment. This difference is due to the fact that the level of investment is a decreasing function of $\theta$ when $\varepsilon 1$, so that the less efficient firms would be imitated by the more efficient ones unless they reduce investment beyond the full information level. (In Besanko and Spulber's model, i.e., with $\varepsilon=0$, investment is a positive function of the cost parameter $\theta$, so that the high cost firms signal their type by investing more than the full information level, therefore alleviating the underinvestment caused by lack of commitment.)

\section{Acknowledgements}

This paper was written while I was a graduate student at the University of Illinois at Champaign-Urbana. I would like to thank Lanny Arvan, Pablo T. Spiller, and an anonymous referee for useful comments and suggestions. This research was partially supported with Fellowship F06701 of the Organization of American States.

\section{References}

Baron, David P., and David Besanko, 1987. "Commitment and Fairness in a Dynamic Regulatory Relationship." Review of Economic Studies 50:413-436.

Baron, David P., and Roger B. Myerson, 1982. "Regulating a Monopolist With Unknown Costs." Econometrica 50(4):911-930.

Besanko, David, and Daniel F. Spulber, 1989. "Sequential Equilibrium Investment by Regulated Firms." Working Paper presented at the Seminar on The Political Economy of Institutions, University of Illinois.

Besanko, David, and Daniel F. Spulber, 1992. "Sequential Equilibrium Investment by Regulated Firms." Rand Journal of Economics 23(2):153-170.

Cho, In-Koo, and David M. Kreps, 1987. "Signaling Games and Stable Equilibria." Quarterly Journal of Economics CII:179-221.

Laffont, Jean-Jacques, and Jean Tirole, 1988. "The Dynamics of Incentive Contracts." Econometrica 56(5):1153-1175. 
Loeb, Martin, and Wesley Magat, 1979. "A Decentralized Method for Utility Regulation." Journal of Law and Economics 22:399-404.

Milgrom, Paul, and John Roberts, 1982. "Limit Pricing and Entry Under Incomplete Information: An Equilibrium Analysis." Econometrica 50(2):443-458.

Kreps, David M., and Robert Wilson, 1982. "Sequential Equilibria." Econometrica 50(4):863-894.

Kydland, Finn E., and Edward C. Prescott, 1977. "Rules Rather than Discretion: The Inconsistency of Optimal Plans." Journal of Political Economy 85(3):619-637.

Williamson, Oliver E. 1975. Markets and Hierarchies: Analysis and Antitrust Implications. New York: Free Press. 